\title{
Successful Treatment of Driveline Infection with Vacuum-Assisted Closure Therapy and Instillation Therapy
}

\author{
Juliane Kilo ${ }^{1}$ Julia Dumfarth ${ }^{1}$ Daniel Höfer ${ }^{1}$ Michael Grimm ${ }^{1}$ \\ ${ }^{1}$ Department of Cardiac Surgery, Regional University Innsbruck, \\ Innsbruck, Austria \\ Address for correspondence Dr Juliane Kilo, Department of Cardiac \\ Surgery, Regional University Innsbruck, Anichstr. 35, Innsbruck 6020, \\ Thorac Cardiovasc Surg Rep 2020;9:e29-e32. \\ Austria (e-mail: juliane.kilo@tirol-kliniken.at).
}

\begin{abstract}
Keywords

- circulatory assist devices

- wound infection

- surgery

- complications

Background Driveline infection is a serious complication in left ventricular assist device (LVAD) patients. We report the case of a patient who was successfully treated by combining instillation and vacuum-assisted closure (VAC) therapy.

Case Description A 65-year-old LVAD patient presented with recurrent driveline infection. Local therapy with VAC therapy in combination with instillation of polyhexanide was performed for 2 weeks. The patient remains free from infection for twelve months by now.

Conclusion This case is the first to present the combination of polyhexanide instillation with VAC as treatment for driveline infection. This therapy may thus be an option for patients who lack any other surgical option.
\end{abstract}

\section{Introduction}

Driveline infection is a serious complication in patients with left ventricular assist device (LVAD) and a major cause of morbidity and mortality.

\section{Case Description}

We report the case of a 65 -year-old patient who underwent LVAD implantation (Heart Ware HVAD [Medtronic, Minneapolis, United States]) due to ischemic cardiomyopathy in November 2016. Relevant comorbidities include renal insufficiency requiring peritoneal dialysis.

One year after LVAD implantation, the patient presented in septic state with high fever and elevated inflammatory parameters (C-reactive protein, $16 \mathrm{mg} / \mathrm{dL}$, leukocytes 17G/L). Staphylococcus aureus could be identified in consecutive blood cultures, as well as from the peritoneal catheter. During the initial critical period the patients' status deteriorated rapidly and the patient suffered from serious right ventricular dysfunction, requiring inotropic support and admission to the intensive care unit. As indicated in - Fig. 1, driveline infection initially presented as massive swelling of the subcutaneous tunnel with a clean entrance site.

Therefore, the driveline tunnel was surgically opened and vacuum assisted closure (VAC) therapy (KCI, Acelity, San Antonio, Texas, United States) was initiated. Simultaneously, the peritoneal catheter was explanted and peritonitis was proven laparoscopically. Microbiologically S. aureus was identified at the surgical site and antibiotic treatment consisted of vancomycin, tigecycline, and meropenem.

The VAC dressing was changed every third day until the swabs returned sterile. After 22 days of vacuum treatment, surgical closure of the wound was performed and transfer to the normal ward was possible 5 days later. The patient was discharged home after a total hospital stay of 55 days.

Four months later, the patient was readmitted to hospital due to recurrence of the driveline infection (- Fig. 2). In a first step, the wound was reopened, debrided, and negative pressure therapy was initiated again. After that, further surgical options were evaluated. Since the patient did not qualify for heart transplantation and neither explantation of the assist device nor omentum plasty were surgical options, we decided to change the conventional vacuum therapy to a combination received

September 3, 2019

accepted

December 22, 2019
DOI https://doi.org/

10.1055/s-0040-1713731. ISSN 2194-7635. (c) 2020 Georg Thieme Verlag KG
Stuttgart · New York

License terms

(๑) $\Theta \circledast$ 


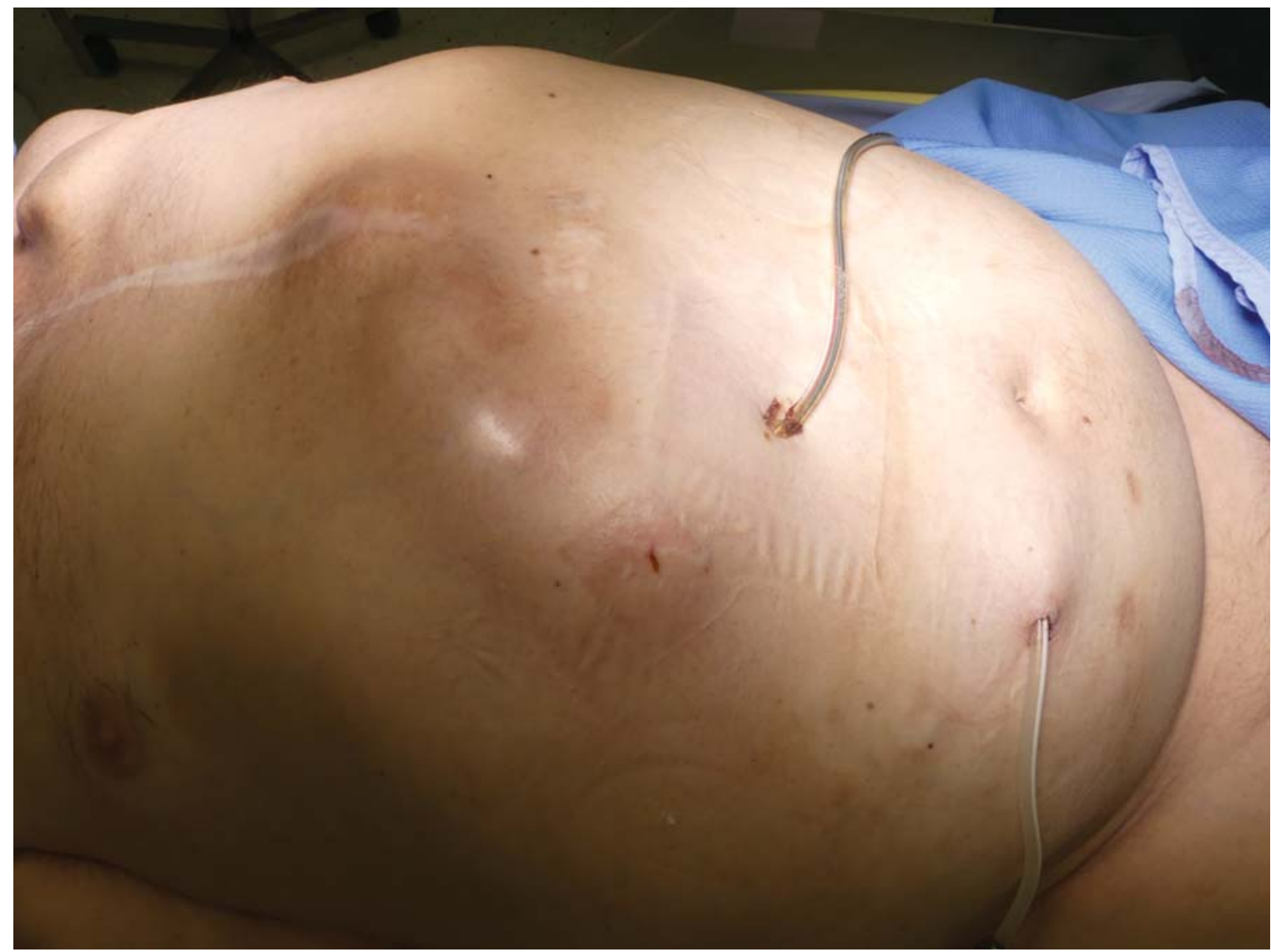

Fig. 1 Initial presentation with infected driveline and peritoneal dialysis catheter.

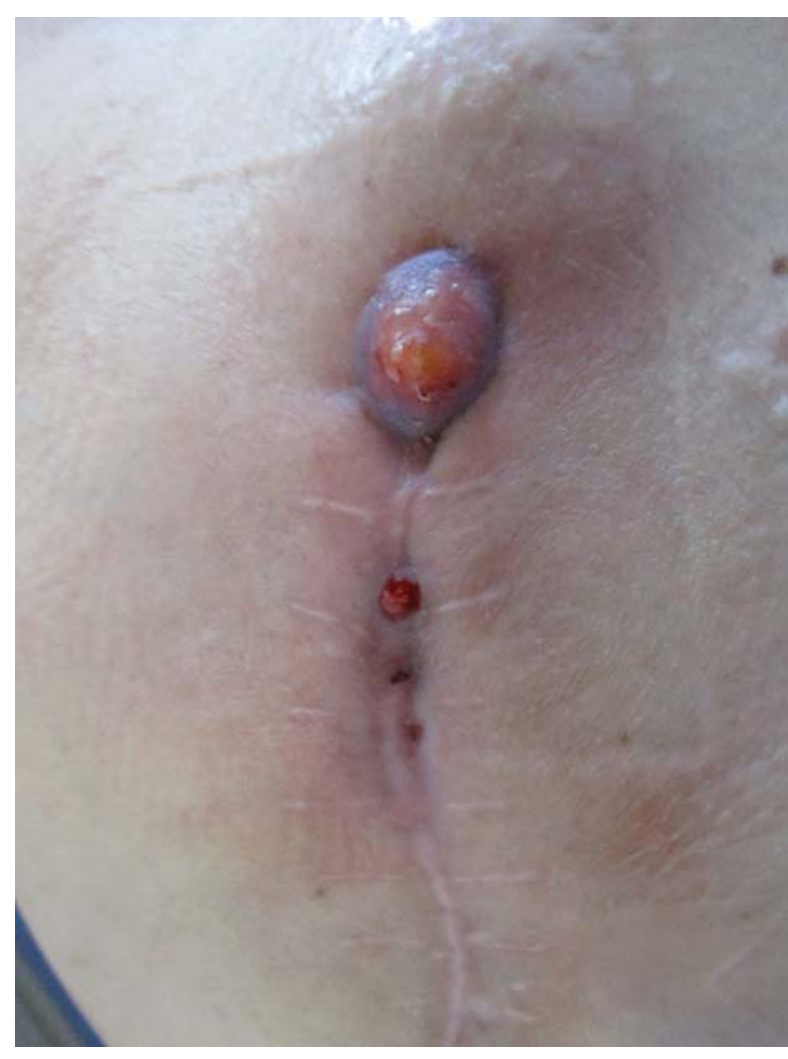

Fig. 2 Recurrence of driveline infection. of vacuum therapy with instillation (VAC Veraflo, KCI Acelity, San Antonio, TX). As detergent, we decided for a $0.04 \%$ polyhexanide solution (Lavasorb, FreseniusKabi, Bad Homburg, Germany) which was installed for 20 minutes into the wound followed by vacuum treatment for 3 hours. Changes of dressing, as well as local debridement, as appropriate, was performed in the operation room without general anesthesia in every 3 days. After 14 days of instillation therapy and sterile swabs, surgical closure of the wound was performed. Postoperatively, the wound was treated with epicutaneous negative pressure wound therapy for another 14 days. Antibiotic treatment was tazobactam/piperacillin and rifampicin. The patient was discharged after a total hospital stay of 31 days. The patient remains free from signs of infection for 12 months by now (-Fig. 3).

\section{Discussion}

Driveline infection is a serious complication after implantation of a LVAD and significantly contributes to mortality. ${ }^{1}$ In patients, who are intended for heart transplantation, driveline infection is a reason for urgent transplantation, although initial results are inferior. ${ }^{2}$

Further surgical alternatives comprise explant of the infected device, relocation implant, ${ }^{3}$ or omentum plasty. ${ }^{4}$ However, all these procedures are major surgical interventions routinely not applicable for patients in seriously impaired 


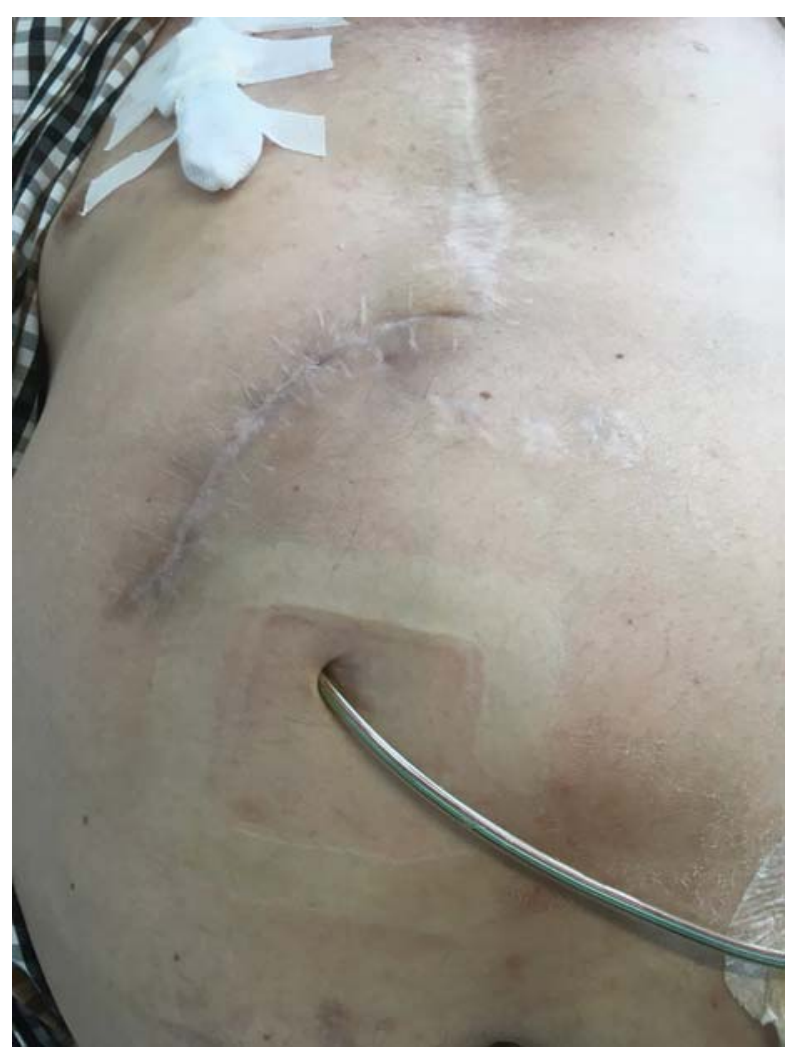

Fig. 3 Situs 9 months after VAC Veraflo Therapy. VAC, vacuumassisted closure. clinical state. In our patient, left ventricular function did not recover during LVAD therapy and therefore device explantation was not considered at any point. Furthermore, omentum plasty was not possible because of the preceding peritonitis, and relocation of the driveline was not considered as an option due to the lack of space of the infection to the pump. In contrast to the most usual cases of driveline infections, which are related to the entrance site, our patient suffered from transperitoneal infection, thus rendering this case unique. Never before has this been described in the literature, although one case reports drainage of ascites via the driveline tunnel. ${ }^{5}$

Negative pressure wound therapy has found its way into clinical routine in the 1990s and indications have been extended ever since. Today, vacuum assisted wound therapy is a standard treatment for surgical wound infections. In cardiac surgery, VAC therapy is used for superficial wound infections at the sternum, as well as on graft harvest sites, deep sternal wound infections, and for postoperative mediastinitis. ${ }^{6}$ Even though literature is sparse concerning vacuum treatment of driveline infection, some cases have been identified, reporting treatment of LVAD infection with VAC therapy. ${ }^{4,7}$ As our department has a large experience in using VAC therapy, we initially treated our patient with surgical opening of the tunnel, local debridement, and negative pressure wound therapy.

Since driveline infection recurred, we had to acknowledge that a new treatment strategy had to be defined. Therefore and because of the lack of any other surgical option, we

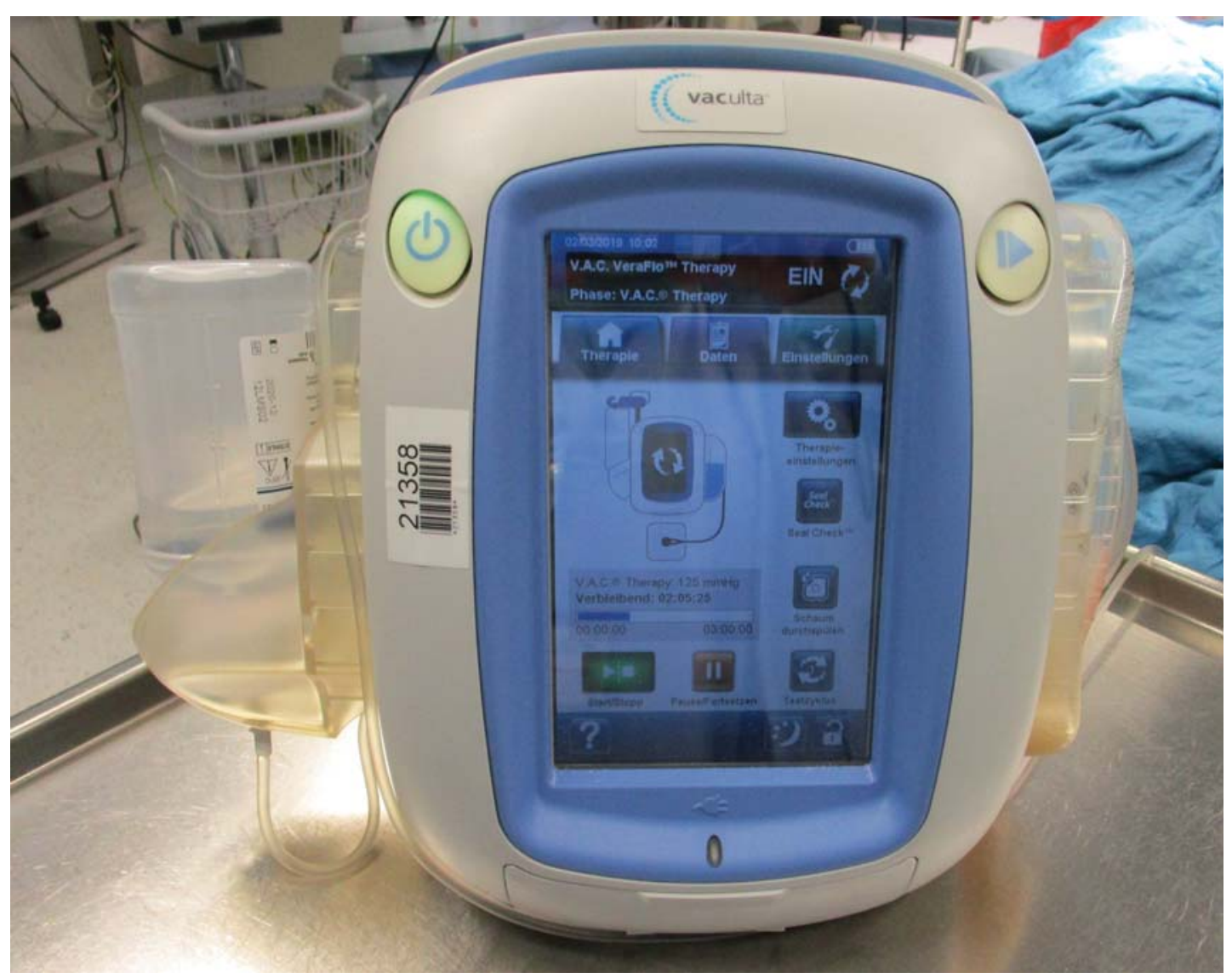

Fig. 4 VAC Veraflo system. VAC, vacuum-assisted closure. 
decided to change our treatment strategy to the combination of instillation and vacuum therapy (VAC Veraflo). The VAC Veraflo is a relatively new therapy, which is not only used for treatment of conventional wounds but especially in orthopaedic surgery. Here it has found its place for conservation of infected implants, ${ }^{8}$ especially in spinal surgery.

One of the major problems, in the treatment of infection of implants, is the lack of susceptibility of antibiotics to penetrate the biofilm of bacteria on the implant.

Penetration of biofilm has been reported for anti-infective solutions. Approved products in Austria include polyhexanide or octenidine. In contrast to the octenidine-based product, Lavasorb is bottled with an infusion port, thus rendering it most useful for instillation therapy (-Fig. 4). The only further case in the literature uses instillation of household bleach (sodium hypochlorite $0.5 \%$ ), which is not approved for use in Austria. ${ }^{9}$ Furthermore, sodium hypochlorite is, not recommended by the manufacturer, because it potentially damages the driveline as well as alcoholic solutions do.

Thus, the combination of VAC therapy and instillation of a locally acting antimicrobial solution seems to be a promising option for the treatment of implant infection.

In our patient, we successfully used the VAC Veraflo therapy with intermittent instillation of $0.04 \%$ polyhexanide solution.

This is the first case to report a successful treatment of recurrent driveline infection with VAC Veraflo therapy.

\section{Conclusion}

In conclusion, we report the first case of successful treatment of driveline infection with VAC Veraflo therapy with polyhexanide. The combination of negative pressure wound therapy and instillation of a local antimicrobial solution is a valuable option for the treatment of patients who lack other surgical options.
Conflict of Interest

None declared.

\section{References}

1 Pavlovic NV, Randell T, Madeira T, Hsu S, Zinoviev R, Abshire M. Risk of left ventricular assist device driveline infection: a systematic literature review. Heart Lung 2019;48(02):90-104

2 Chahal D, Sepehry AA, Nazzari H, Wright AJ, Toma M. The impact of left ventricular assist device infections on postcardiac transplant outcomes: a systematic review and meta-analysis. ASAIO J 2019;65(08):827-836

3 Kurihara C, Nishimura T, Kinoshita O, et al. Successful treatment of mediastinitis after ventricular assist device implantation with rerouting of the outflow vascular prosthesis. J Artif Organs 2011; 14(02):155-158

4 Kimura M, Nishimura T, Kinoshita O, et al. Successful treatment of pump pocket infection after left ventricular assist device implantation by negative pressure wound therapy and omental transposition. Ann Thorac Cardiovasc Surg 2014;20(Suppl):842-845

5 Walter V, Stock UA, Soriano-Romero M, Schnitzbauer A, Moritz A, Beiras-Fernandez A. Eradication of a chronic wound and driveline infection after redo-LVAD implantation. J Cardiothorac Surg 2014; 9:63

6 Yu AW, Rippel RA, Smock E, Jarral OA. In patients with poststernotomy mediastinitis is vacuum-assisted closure superior to conventional therapy? Interact Cardiovasc Thorac Surg 2013;17 (05):861-865

7 Baradarian S, Stahovich M, Krause S, Adamson R, Dembitsky W. Case series: clinical management of persistent mechanical assist device driveline drainage using vacuum-assisted closure therapy. ASAIO J 2006;52(03):354-356

8 Lehner B, Fleischmann W, Becker R, Jukema GN. First experiences with negative pressure wound therapy and instillation in the treatment of infected orthopaedic implants: a clinical observational study. Int Orthop 2011;35(09):1415-1420

9 Idris A, Hart A, Branam S, Guglin M. VeraFlo negative pressure wound therapy with household bleach for refractory driveline infection in a patient with left ventricular assist device. VAD J 2015;1:1-10 